\title{
Linear Multistep Methods for Volterra Integral and Integro-Differential Equations
}

\author{
By P. J. van der Houwen and H. J. J. te Riele
}

\begin{abstract}
A general class of linear multistep methods is presented for numerically solving firstand second-kind Volterra integral equations, and Volterra integro-differential equations. These so-called VLM methods, which include the well-known direct quadrature methods, allow for a unified treatment of the problems of consistency and convergence, and have an analogue in linear multistep methods for ODEs, as treated in any textbook on computational methods in ordinary differential equations.

General consistency and convergence results are presented (and proved in an Appendix), together with results of numerical experiments which support the theory.
\end{abstract}

1. Introduction. We consider Volterra integral equations of the form

$$
\theta y(t)=g(t)+\int_{t_{0}}^{t} K(t, \tau, y(\tau)) d \tau, \quad t \in I:=\left[t_{0}, T\right], \theta=0,1 .
$$

This equation is called of the first kind if $\theta=0$ and of the second kind if $\theta=1$. Furthermore, we consider Volterra integro-differential equations

$$
\frac{d y}{d t}=f(t, y(t), z(t)), \quad z(t)=g(t)+\int_{t_{0}}^{t} K(t, \tau, y(\tau)) d \tau, \quad t \in I,
$$

where $y\left(t_{0}\right)=y_{0}$. In these equations $y(t)$ is the unknown function and $g, K$ and $f$ are given, nonsingular functions on $I, S \times \mathbf{R}$ and $I \times \mathbf{R} \times \mathbf{R}$, respectively, where $S:=\left\{(t, \tau), t_{0} \leqslant \tau \leqslant t \leqslant T\right\}$. The conditions which ensure the existence of a unique, continuous solution of (1.1) and (1.2) are listed in Appendix I. (All appendices appear in the supplements section at the end of this issue.)

There exists a vast amount of literature dealing with the numerical solution of (1.1) and (1.2), and dealing with the many physical applications which underly these equations ([1], [16], [18]).

A common, simple way of solving (1.1) numerically is obtained by writing these equations down in a sequence of equidistant points

$$
t_{n}:=t_{0}+n h, \quad n=0(1) N, h \text { fixed and } t_{N}=T,
$$

approximating the integral term by some suitably chosen quadrature formula, and solving the resulting equation for $y\left(t_{n}\right)$ successively for $n=n_{0}(1) N$, where $n_{0}$ is some suitable starting index. Equation (1.2) is commonly solved by integrating the

Received April 11, 1983; revised May 14, 1984.

1980 Mathematics Subject Classification. Primary 65R20.

Kev words and phrases. Numerical analysis, Volterra integral and integro-differential equations, linear multistep methods, consistency, convergence. 
differential equation in the points (1.3) (say), using an LM formula for ODEs, $z\left(t_{j}\right)$ being approximated by some suitably chosen quadrature formula. All these methods are called direct quadrature (DQ) methods. DQ methods may give satisfactory results, but sometimes the results with DQ are completely worthless, as was demonstrated for first-kind equations by Linz [12, p. 67], who applied a fourth-order Gregory quadrature method to the very simple integral equation

$$
\begin{aligned}
& 0=-\sin t+\int_{0}^{t} \cos (t-\tau) y(\tau) d \tau \\
& I=[0,2], \text { with exact solution } y(t)=1 .
\end{aligned}
$$

The "approximate" values obtained for $y(2)$ were 8.4 and $1.5 \times 10^{7}$ for $h=0.1$ and $h=0.05$, respectively. For second-kind equations, too, the Gregory rules will fail if large Lipschitz constants for the kernel function with respect to $y$ are involved (see Example 2.4.2).

In this paper we present a general class of linear multistep methods for (1.1) and (1.2) which includes the DQ methods. (It should be remarked that such methods for second-kind Volterra integral equations were already introduced in [9], and results were presented without proof.) A characteristic feature of this class is that it involves linear combinations, not only of $y$ - and $K$-values, but also of values of the auxiliary function (called the lag term)

$$
Y(t, s):=g(t)+\int_{t_{0}}^{s} K(t, \tau, y(\tau)) d \tau
$$

for $(t, s) \in S$. Note that we may write (1.1) as $\theta y(t)=Y(t, t)$.

This general class will be called Volterra linear multistep (VLM) methods. VLM methods allow for a uniform treatment of the problems of consistency and convergence, and have an analogue in linear multistep methods for ordinary differential equations, as treated, e.g., by Lambert in [11].

In Section 2 of this paper we treat VLM methods for Volterra integral equations of the second and of the first kind jointly. We motivate the introduction of the general class of VLM methods; examples of VLM methods are presented and consistency and convergence results for VLM methods are derived. Numerical experiments with several examples of VLM methods are reported. In a similar way as is done in Section 2, Section 3 treats VLM methods for integro-differential equations. It turns out that several results of Section 2 for second-kind Volterra integral equations can be used in Section 3. The proofs of the theorems presented in Sections 2 and 3 are given, as far as they are nontrivial, in Appendix IV to this paper.

\section{VLM methods for Volterra Integral Equations.}

\subsection{The General VLM Method.}

2.1.1. The family of $D Q$ methods. In order to state our general VLM method for (1.1) we introduce numerical approximations $y_{n}$ to $y\left(t_{n}\right)$ and $Y_{n}(t)$ to $Y\left(t, t_{n}\right)$, and we let

$$
K_{n}(t):=K\left(t, t_{n}, y_{n}\right), \quad n \geqslant 0 .
$$

We assume that $Y_{n}(t), t \geqslant t_{n}$, will be computed by a quadrature formula of the form

$$
Y_{n}(t):=g(t)+h \sum_{j=0}^{n} w_{n j} K_{j}(t), \quad n \geqslant n_{1},
$$


where the $w_{n j}$ are given weights and $n_{1}$ is sufficiently large in order to ensure the required order of accuracy. When we say that the order of this quadrature formula is $r$, we mean that for any $t \geqslant t_{n}$

$$
E_{n}(h ; t):=\int_{t_{0}}^{t_{n}} K(t, \tau, y(\tau)) d \tau-h \sum_{j=0}^{n} w_{n j} K\left(t, t_{j}, y\left(t_{j}\right)\right)=\mathcal{O}\left(h^{r}\right)
$$

as $h \rightarrow 0, n \rightarrow \infty$, with $t_{n}=t_{0}+n h$ fixed. An important class of quadrature formulas, which includes the well-known Gregory formulas, are the so-called $(\rho, \sigma)$ reducible quadrature formulas [20]. However, except for the Gregory formulas (which can be traced back to the Adams-Moulton (AM) formulas), these quadrature formulas give rise to rather awkward implementations. Hence, from a computational point of view we would like to approximate the lag term by means of a Gregory rule. This will be the case in all our numerical experiments.

The (conventional) DQ method for (1.1) mentioned in the introduction is a direct discretization of the (equivalent) equation $\theta y(t)=Y(t, t)$, viz.

$$
\theta y_{n}=Y_{n}\left(t_{n}\right), \quad n=n_{1}(1) N .
$$

2.1.2. Motivation for extending the family of $D Q$ methods. It has already been observed that DQ methods may fail,both for first- and second-kind equations, when Gregory rules are used for approximating the lag term. To overcome this unfavorable behavior, methods have been proposed in which the effect of the lag term approximation is reduced.

If $\theta=0$ (first-kind equations), an often applied remedy (cf. [1, p. 898], see also [12]) may be the differentiation of Eq. (1.1) to obtain the (implicit) second-kind equation (assuming that $g^{\prime}$ and $K_{t}$ exist)

$$
g^{\prime}(t)+\int_{t_{0}}^{t} K_{t}(t, \tau, y(\tau)) d \tau=-K(t, t, y(t))
$$

or more compactly,

$$
Y_{t}(t, t)=-K(t, t, y(t)) .
$$

If $K$ is linear in $y: K(t, t, y)=K^{*}(t, t) y$, with $K^{*}(t, t) \neq 0$, then (2.1.5) may be solved by standard DQ methods for second-kind equations. If the derivatives involved in (2.1.5) cannot be evaluated analytically, or if $K$ or $g$ are given in tabular form, $Y_{t}$ may be replaced by a $k$-step forward difference approximation (see Appendix II. Notice that the order of the DQ method used for solving (2.1.5) is not affected by this approximation, provided that the order of this difference approximation is at least as large as the order of the DQ method; cf. [12, pp. 81-82]). In that case, this equation can be approximated by

$$
\sum_{j=0}^{k} \delta_{j} Y\left(t_{n+j}, t_{n}\right)=h K\left(t_{n}, t_{n}, y\left(t_{n}\right)\right), \quad t_{n}:=t_{0}+n h .
$$

Finally, approximating $Y\left(t, t_{n}\right)$ by the direct quadrature formula (2.1.2) leads to

$$
\sum_{j=0}^{k} \delta_{j} Y_{n}\left(t_{n+j}\right)=h K_{n}\left(t_{n}\right)
$$


(The difference with (2.1.4a, $\theta=0)$ is that now both $Y$ and $K$ are involved.) Here, it is assumed that $K(t, t, y(t))$ does not vanish. Otherwise, (2.1.5) is again a first-kind equation and the process described above can be repeated to obtain a formula similar to (2.1.4b) in the sense that linear combinations of $Y_{n}$ and $K_{n}$ values are involved.

If $K(t, t, y(t))$ is small, then (2.1.4b) may produce poor results because it is in fact a direct quadrature method for a stiff second-kind equation. To see this more clearly, let $K$ be linear: $K(t, s, y)=K^{*}(t, s) y$; then (2.1.5) can be written in the form (1.1) with $\theta=1$ and with the kernel $-K_{t}^{*}(t, s) y / K^{*}(t, t)$, that is, an equation of the second kind in which the kernel has a large Lipschitz constant with respect to $y$. Approximating the derivatives in this stiff equation numerically and applying the DQ method leads to formulas of the type (2.1.4b). When using Gregory rules, such methods are poorly stable because of the restricted stability regions of the underlying AM formulas.

The above considerations prompt us to look for alternatives of the DQ method for stiff, second-kind equations. Following [8], we differentiate (1.1) to obtain the integro-differential equation

$$
y^{\prime}(t)=K(t, t, y(t))+Y_{t}(t, t)
$$

Approximating $Y_{t}(t, t)$ by the $k$-step forward differentiation formula and then applying a linear $k$-step method to the resulting ODE, we obtain the formula

$$
\sum_{i=0}^{k} a_{i} y_{n-i}+\sum_{i=0}^{k} \sum_{j=-i}^{k-i} b_{i} \delta_{i+j} Y_{n-i}\left(t_{n+j}\right)=h \sum_{i=0}^{k} b_{i} K_{n-i}\left(t_{n-i}\right)
$$

Again, the lag term does not play the central role in this formula as it does in formula $(2.1 .4 \mathrm{a}, \theta=1)$. In particular, if $Y(t, s)$ does not depend on $t$ the whole expression containing the lag terms vanishes in $(2.1 .4 \mathrm{c})$.

Thus, the formulas $(2.1 .4 \mathrm{a})-(2.1 .4 \mathrm{c})$ are all relations exhibiting some linear combination of $y_{i}{ }^{-}, Y_{i}\left(t_{j}\right)$ - and $K_{i}\left(t_{j}\right)$-values. This leads us to consider a general class of Volterra Linear Multistep (VLM) methods in which numerical approximations to $y(t), Y(t, \tau)$ and to $K(t, \tau, y(\tau))$ occur linearly in a set of points in the $(t, \tau)$-plane as indicated in Figure 2.1.1.

2.1.3. Definition of VLM methods. Our general VLM method for (1.1) consists of

(i) the VLM formula

$$
\begin{aligned}
& \theta \sum_{i=0}^{k} \alpha_{i} y_{n-i}+\sum_{i=0}^{k} \sum_{j=-k}^{k} \beta_{i j} Y_{n-i}\left(t_{n+j}\right) \\
& \quad=h \sum_{i=0}^{k} \sum_{j=-k}^{k} \gamma_{i j} K_{n-i}\left(t_{n+j}\right), \quad n=k^{*}(1) N
\end{aligned}
$$

( $k^{*}$ fixed), where $\alpha_{i}, \beta_{i j}$ and $\gamma_{i j}, i=0(1) k, j=-k(1) k$, are to be prescribed, and

(ii) the lag term formula (2.1.2) for the computation of $Y_{n-i}\left(t_{n+j}\right)$.

In the VLM method the quantities $y_{1}, \ldots, y_{k^{*}-1}$ with $k^{*}=k+n_{1}$ are assumed to be 


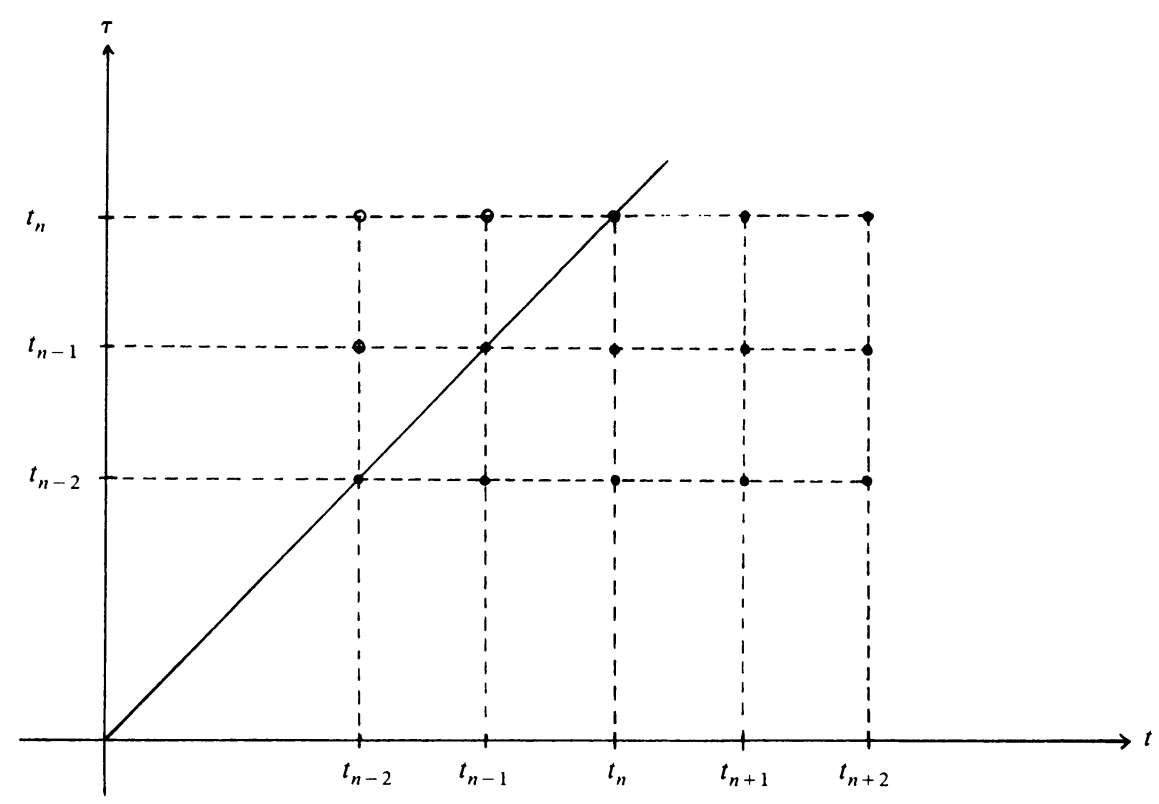

FIGURE 2.1.1

Points in the $(t, \tau)$-plane needed in (2.1.4) for $k=2$

precomputed by some starting method. Then $y_{k^{*}}, \ldots, y_{N}$ can be successively computed using (2.1.4). Since the kernel $K(t, \tau, y)$ is not necessarily defined outside $S$, we require $\beta_{i j}=\gamma_{i j}=0$ for $i+j<0$. In addition, we will only consider VLM methods where $\beta_{i j}=\gamma_{i j}=0$ for $i+j>k$. Furthermore, if $\beta_{i j}, \gamma_{i j} \neq 0$ for $j=1(1) k$, we assume that the domain of definition of $K$ can be extended to points $(t, \tau)$ with $t \leqslant T+k h, \tau \leqslant T$. It is convenient to characterize (2.1.4) by the matrices

$$
A=\left(\alpha_{i}\right), \quad B=\left(\beta_{i j}\right), \quad C=\left(\gamma_{i j}\right)
$$

where the row index $i$ assumes the values $0(1) k$ and the column index $j$ the values $-k(1) k$ (see Figure 2.1.1: the point $\left(t_{n+j}, t_{n-i}\right)$ corresponds to the matrix elements $\beta_{i j}$ and $\gamma_{i j}$ ). Note that for $\theta=0$ the values of the coefficients $\alpha_{i}$ in (2.1.4) are irrelevant. We will be particularly interested in VLM formulas with vanishing row sums in the $B$ matrix, in order to reduce the effect of the lag term approximation.

It should be remarked that in the theory of Volterra-Runge-Kutta (VRK) methods we have a similar situation as for the VLM methods described here; that is, the VRK method consists of a $V R K$ formula, involving (nonlinearly) the lag term and the kernel function at a number of step and nonstep points in the $(t, s)$-plane, and a quadrature rule for approximating the lag term [4].

2.1.4. Special families of VLM methods.

1. Direct quadrature methods. Direct quadrature methods for (1.1) are characterized by the $(1 \times 1)$ matrices $A=I, B=-I, C=0$, for which (2.1.4) reduces to the simple scheme (2.1.4a). 
2. Indirect linear multistep methods. The formula (2.1.4c) is of the form (2.1.4) and is generated by the matrices

$$
A=\left[\begin{array}{c}
a_{0} \\
a_{1} \\
\vdots \\
a_{k}
\end{array}\right], \quad B=\left[\begin{array}{ccccccc} 
& & & b_{0} \delta_{0} & \cdots & b_{0} \delta_{k-1} & b_{0} \delta_{k} \\
& \bigcirc & b_{1} \delta_{0} & b_{1} \delta_{1} & \cdots & b_{1} \delta_{k} & \\
& . & & \vdots & . & \bigcirc \\
b_{k} \delta_{0} & & \cdots & & b_{k} \delta_{k} & &
\end{array}\right],
$$

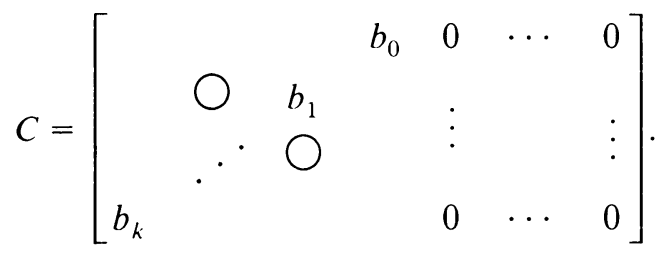

This VLM formula forms, together with (2.1.2), a so-called indirect linear multistep (ILM) method for (1.1), not only for $\theta=1$, but also for $\theta=0$. When the $a_{i}$ and $b_{i}$ are the coefficients of a backward differentiation method (for $k=1(1) 5$ these are listed in Table 2 of Appendix II), (2.1.4) represents the so-called IBD (indirect backward differentiation) formula. For this method, we have $b_{0}=1, b_{>0}=0$ and $b_{0} \delta_{j}=a_{j}, j=0(1) k$.

As was shown in [8] for $\theta=1$, the IBD methods have excellent stability properties, irrespective of the lag term formula chosen (see also the numerical experiments in Section 2.4).

Notice that formula (2.1.4b) (which is often recommended for first-kind equations when the DQ approach diverges) is in fact the IBD formula with $\theta=0$.

It should be remarked that the ILM methods require the extension of the domain of definition $S$ with the points $\left\{(t, \tau) \mid T<t \leqslant T+k h, t_{0} \leqslant \tau \leqslant T\right\}$. In this connection we observe that if $S$ can also be extended to points with $t<\tau$, we may use backward instead of forward differentiation coefficients $\delta_{i}$ in the IBD method, i.e., the matrix $B$ is replaced by the matrix $\left(\beta_{i j}\right)$ all elements of which vanish, except for those in the first row, which are given by $\left(b_{0} \boldsymbol{\delta}_{k}, b_{0} \boldsymbol{\delta}_{k-1}, \ldots, b_{0} \boldsymbol{\delta}_{0}, 0, \ldots, 0\right)$.

3. Multilag methods. In [22], [23] we find methods for (1.1) with $\theta=1$ which can be characterized by the matrices (by $\bigcirc_{k}$ we mean the $(k \times k)$ null-matrix)

(2.1.9) $A=\left[\begin{array}{c}a_{0} \\ 0 \\ \vdots \\ 0\end{array}\right], \quad B=\left[\begin{array}{ccccc}0 & \cdots & 0 & \cdots & 0 \\ & a_{1} & & \\ \bigcirc_{k} & \vdots & \bigcirc_{k}\end{array}\right], \quad C=\left[\begin{array}{ccccc}0 & \cdots & b_{0} & \cdots & 0 \\ & b_{1} & & \\ & & & \end{array}\right]$.

These methods were called multilag methods (ML) for (1.1) with $\theta=1$. Here, the $a_{i}$ and $b_{i}, i=0(1) k$, may be the coefficients of any LM method for ODEs. Wolkenfelt has pointed out that in the case that the lag term $Y_{n}(t)$ is computed by using a quadrature rule which is reducible to an LM-method for ODEs with the same coefficients $a_{i}$ and $b_{i}$, then the resulting method is, in fact, equivalent to a DQ method based on the same quadrature rule (provided, of course, that identical starting values are used). 
4. Modified multilag methods. In [22] Wolkenfelt introduced a modification of the ML methods, viz., the so-called modified multilag (MML) methods for (1.1), characterized by the matrices

(2.1.10) $A=\left[\begin{array}{c}a_{0} \\ a_{1} \\ \vdots \\ a_{k}\end{array}\right], \quad B=\left[\begin{array}{ccccc}0 & \cdots & 0 & \cdots & 0 \\ \bigcirc & -a_{1} & a_{1} & \\ . & \bigcirc & \vdots & \bigcirc_{k} \\ -a_{k} & & a_{k} & \end{array}\right], \quad C=\left[\begin{array}{ccccc}0 & \cdots & b_{0} & \cdots & 0 \\ & b_{1} & \\ \bigcirc_{k} & \vdots & \bigcirc_{k} \\ & b_{k} & \end{array}\right]$

The $a_{i}$ and $b_{i}$ are, again, the coefficients of any LM method for ODEs. A common choice are the Adams-Moulton formulas (listed in Table 3 of Appendix III, for $k=1(1) 5)$. As with ML methods, the MML method is algebraically equivalent to the DQ method if in both methods the lag term formula is based on the LM formula $\left\{a_{i}, b_{i}\right\}$ and if the starting values would be identical, where $y_{n}=Y_{n}\left(t_{n}\right)$ for $n_{1} \leqslant n$ $\leqslant n_{1}+k$. However, the crucial difference with the ML methods is that by virtue of the vanishing row sums in the $B$ matrix, the stability of MML formulas is hardly influenced by the lag term formula, whereas the stability of ML methods largely depends on the lag term formula. Since lag term formulas other than the (poorly stable) Gregory formulas give rise to rather awkward implementations, the MML methods may be more efficient than the DQ and ML methods if stability becomes important, that is, if large $\partial K / \partial y$ values are involved (see Example 2.4.3).

2.2. Consistency of VLM Methods for Volterra Integral Equations. As is usual in the consistency analysis of numerical schemes for functional equations, we substitute the exact solution $y(t)$ of (1.1) into (2.1.4), and analyze the resulting residue. With the relations $\theta y(t)=Y(t, t)$ and $\partial Y(t, s) / \partial s=K(t, s, y(s))$, and (2.1.3), we obtain

$$
\begin{gathered}
\sum_{i=0}^{k}\left\{\theta \alpha_{l} y\left(t_{n-i}\right)+\sum_{j=-k}^{k}\left(\beta_{i j}\left[g\left(t_{n+j}\right)+h \sum_{l=0}^{n-i} w_{n-i, l} K\left(t_{n+j}, t_{l}, y\left(t_{l}\right)\right)\right]\right.\right. \\
\left.\left.-h \gamma_{i j} K\left(t_{n+j}, t_{n-i}, y\left(t_{n-i}\right)\right)\right)\right\} \\
=\mathbf{L}_{n}[Y]+\mathbf{Q}_{n}[y],
\end{gathered}
$$

where we have introduced the operators $\mathbf{L}_{n}: C_{2}^{1}(S) \rightarrow \mathbf{R}$ and $\mathbf{Q}_{n}: L^{1}(\mathbf{R}) \rightarrow \mathbf{R}$,

$$
\begin{gathered}
\mathbf{L}_{n}[Y]:=\sum_{i=0}^{k}\left\{\boldsymbol{\theta} \alpha_{i} Y\left(t_{n-i}, t_{n-i}\right)+\sum_{j=-k}^{k}\left[\beta_{i j}-\gamma_{i j} h \frac{\partial}{\partial s}\right] Y\left(t_{n+j}, t_{n-i}\right)\right\} \\
\mathbf{Q}_{n}[y]:=-\sum_{i=0}^{k} \sum_{j=-k}^{k} \beta_{i j} E_{n-i}\left(h ; t_{n+j}\right) .
\end{gathered}
$$

Here, $C_{2}^{1}(S)$ denotes the space of continuous functions $Y(t, s)$, differentiable with respect to $s$ for all $(t, s) \in S$.

The residue (2.2.1) will be called the local truncation error of the VLM method. It consists of two terms: the first term is determined by the VLM formula (2.1.4) and will be called the local truncation error of the VLM formula; the second term originates from the quadrature formula (2.1.2) approximating the lag term (1.5) and will be called the lag term error. Both errors play a role in the convergence analysis 
of VLM methods; in Section 2.3 we will see that the rate of convergence of the numerical solution to the exact solution is closely related to that of the local truncation error of the method. The convergence of the local truncation error is of ten called consistency:

Definition 2.2.1. (a) The VLM method is said to be consistent with Eq. (1.1) if both the VLM formula and the lag term formula are consistent.

(b) The VLM formula and the lag term formula are said to be consistent if the respective errors $\mathbf{L}_{n}[Y]$ and $\mathbf{Q}_{n}[y] \rightarrow 0$ as $h \rightarrow 0$ where $Y$ and $y$ correspond to the exact solution. They are said to be consistent of order $p$ if the errors are $\mathcal{O}\left(h^{p+1}\right)$ as $h \rightarrow 0$.

For the lag term formula we immediately have that $\mathbf{Q}_{n}[y]=\mathcal{O}\left(h^{r}\right)$ if the lag term quadrature rule is of order $r$ (cf. (2.1.3)).

For the VLM formula the order of its truncation error depends on the parameters $\alpha_{j}, \beta_{i j}$ and $\gamma_{i j}$. By expanding $\mathbf{L}_{n}[Y]$ in a Taylor series about $t_{n}$, conditions can be derived for which $\mathbf{L}_{n}[Y]=\mathcal{O}\left(h^{p+1}\right)$ as $h \rightarrow 0$. However, such an expansion requires sufficient differentiability of the lag term function $Y(t, s)$ with respect to $t$ and $s$. From its definition (1.5) it then follows that the functions $g$ and $K$ and the solution $y$ should be in a class of sufficient differentiability. Since for existence and uniqueness it is only required that $y \in C(I), g \in C^{1-\theta}(I)$ and $K \in C^{1-\theta}(S \times \mathbf{R})$ (see Appendix I), we conclude that generally we cannot expand $\mathbf{L}_{n}[Y]$ in a Taylor series of sufficiently high order. Therefore, and in analogy with the consistency theory for ODEs (see, e.g., Lambert [11, p. 23]), we allow $\mathbf{L}_{n}$ to operate on an arbitrary test function $X$ which we may assume to have as many higher derivatives as we require. In this way we can formally define the order of the VLM formula.

Definition 2.2.2. The operator (2.2.2a) and the associated VLM formula (2.1.4) are said to be of order $p$ if for all $X \in C^{p+1}(S)$, which in the case of first-kind equations $(\theta=0)$ vanish on the line $t=\tau$, we have $\mathbf{L}_{n}[X]=\mathcal{O}\left(h^{p+1}\right)$ as $h \rightarrow 0$ with nonvanishing error constant.

We emphasize that the order conditions for VLM formulas only refer to the VLM formula itself and do not involve the exact solution, whereas the consistency of the VLM formula refers to both the solution and the VLM formula. A similar situation holds for LM and RK methods for ODEs and for VRK methods for Volterra equations.

In the remainder of this section we concentrate on the derivation of the order conditions for VLM formulas and we will not be concerned with lag term approximations.

The following two theorems express the order $p$ conditions, in terms of the parameters occurring in (2.1.4), for (1.1) in the cases $\theta=1$ and $\theta=0$, respectively. The proofs are given in Appendix IV.

THEOREM 2.2.1. If $\theta=1$ then the operator $\mathbf{L}_{n}$ and the associated VLM formula (2.1.4) are of order $p$ if $C_{q l}=0$ for $q=0(1) p, l=0(1) q$, where

$$
C_{q l}:=\frac{1}{(q-l) ! l !} \sum_{i=0}^{k}\left[(-i)^{q} \alpha_{i}-\sum_{j=-k}^{k} j^{q-l}(-i)^{l-1}\left(i \beta_{i j}+l \gamma_{i j}\right)\right]
$$

with the convention that $(-i)^{l-1} l=0$ if $i=l=0$. 
THEOREM 2.2.2. If $\theta=0$ then the operator $\mathbf{L}_{n}$ and the associated VLM formula (2.1.4) are of order $p$ if $B_{q l}=0$ for $q=1(1) p, l=1(1) q$, where

$$
\begin{aligned}
B_{q l}:=\frac{1}{(q-l) ! l !} \sum_{i=0}^{k} \sum_{j=-k}^{k}(j-i)^{q-l-1}(j+i)^{l-1} \\
\cdot\left[\beta_{i j}\left(j^{2}-i^{2}\right)-\gamma_{i j}(q j+q i-2 l j)\right] .
\end{aligned}
$$

The various orders of the illustrating families introduced in Section 2.1.4 can now be found by substituting the relevant values of the parameters $\alpha_{i}, \beta_{i j}$ and $\gamma_{i j}$ into the above two theorems. The results are summarized in the following corollary.

COROLlaRY 2.2.1. Let $\tilde{p}$ be the order of the LM formula for ODEs defining the coefficients $\left\{a_{i}, b_{i}\right\}$ in families 2,3 and 4. Then the order $p$ of the operator $\mathbf{L}_{n}$ and of the associated VLM formula (2.1.4) is given by

$$
p=\left\{\begin{array}{l}
\infty \quad \text { for family } 1(D Q) \\
\min \{k, \tilde{p} / \theta\} \quad \text { for family } 2(\text { ILM }) \\
\tilde{p} \quad \text { both for families } 3 \text { and } 4(M L \text { resp. MML }) .
\end{array}\right.
$$

Since the DQ method is completely determined by the lag term formula the infinite order of the DQ formula is only a formal result. Note that for $\theta=0$ in the ILM case this corollary gives $p=k$, independent of the order $\tilde{p}$, and, in fact, in this case the $\left\{a_{i}, b_{i}\right\}$ need not represent an LM method for ODEs at all.

Instead of starting with an already known VLM formula, such as (M)ML and ILM formulas, one can derive VLM formulas directly from the order conditions. We will illustrate this for second-kind equations $(\theta=1)$ and for the special class where all row sums in the $B$-matrix vanish. First, we consider the order equations $C_{q, q}=0$, i.e.,

$$
\sum_{i=0}^{k}\left[(-i)^{q} \alpha_{i}-(-i)^{q-1} q \gamma_{i}\right]=0, \quad q=0(1) p
$$

where the $\gamma_{i}$ denote the row sums in the $C$ matrix. It is easily verified that these conditions are just the order equations of a $p$ th order LM method for ODEs with coefficients $\left\{\alpha_{i}, \gamma_{i}\right\}$. Thus, by choosing some $p$ th order LM method we prescribe the $\alpha_{i}$ and the row sums in the $C$ matrix. There remain $\frac{1}{2} p(p+1)$ order equations $C_{q, l}=0, l=0(1) q-1, q=1(1) p$. Summarizing, we have $2(k+1)+\frac{1}{2} p(p+1)$ linear equations for the parameters $\beta_{i j}$ and $\gamma_{i j}$. Restricting these parameters to points in the domain of definition (see Figure 2.1.1), we have $(3 k+2)(k+1)$ free parameters. Let us choose a second-order BD method to fix the parameters $\alpha_{i}$ and $\gamma_{i}$. An example of the many possible formulas generated by these $\left\{\alpha_{i}, \gamma_{i}\right\}$ is given by

$$
\begin{aligned}
3 y_{n}-4 y_{n-1}+y_{n-2}+\left[\frac{3}{2} Y_{n}\left(t_{n}\right)\right. & -2 Y_{n}\left(t_{n+1}\right)+\frac{1}{2} Y_{n}\left(t_{n+2}\right)+2 Y_{n-1}\left(t_{n-1}\right) \\
& \left.-2 Y_{n-1}\left(t_{n}\right)-2 Y_{n-2}\left(t_{n-2}\right)+2 Y_{n-2}\left(t_{n}\right)\right] \\
=2 h K_{n}\left(t_{n}\right) . &
\end{aligned}
$$

This formula does not belong to the (M)ML or ILM family.

Let us assume in the rest of this section that the VLM formula (2.1.4) is of order $p$. From the proofs of Theorems 2.2.1, resp. 2.2.2, it follows that the local truncation error $\mathbf{L}_{n}[Y]$ can be expressed in terms of the constants defined in (2.2.3), resp. 
(2.2.4), as follows:

$$
\mathbf{L}_{n}[Y]=h^{p+1} \sum_{l=0}^{p+1} C_{p+1 . l} Y^{(p+1-l . l)}+\mathcal{O}\left(h^{p+2}\right) \quad \text { as } h \rightarrow 0,
$$

resp.

$$
\mathbf{L}_{n}[Y]=h^{p+1} \sum_{l=1}^{p+1} B_{p+1, l} Z^{(p+1-l, l)}+\mathcal{O}\left(h^{p+2}\right) \quad \text { as } h \rightarrow 0
$$

where

$$
\begin{aligned}
Y^{(i, j)} & :=\left.(\partial / \partial t)^{i}(\partial / \partial s)^{j} Y(t, s)\right|_{t=s=t_{n},} \\
Z^{(i, j)} & :=\left.(\partial / \partial u)^{i}(\partial / \partial v)^{j} Z(u, v)\right|_{u=2 t_{n}, v=0}, \\
Z(u, v) & :=Y\left(\frac{u+v}{2}, \frac{u-v}{2}\right)
\end{aligned}
$$

In order to compare the values of the error constants $C_{p+1, l}$ and $B_{p+1, l}$ for the various families introduced in Section 2.1.4, we have evaluated and simplified the expressions for these constants as much as possible, and obtained the following results.

For $\theta=1$, Corollary 2.2.1 gives for the ILM formula: $p=k$ provided that $\tilde{p} \geqslant k$ (which is a reasonable assumption, valid, e.g., when the LM formula for ODEs is a Backward Differentiation formula $(\tilde{p}=k)$ or an Adams-Moulton formula $(\tilde{p}=$ $k+1)$ ). Hence,

$$
C_{p+1, l}=\frac{(-1)^{p-1}}{(p+1-l) ! l !} \sum_{i=0}^{k}\left\{i^{p}\left[i a_{i}+(p+1) b_{i}\right]-R\right\}, \quad p=k,
$$

with $R=k ! b_{i}$ if $l=0$ and $R=0$ if $l=1,2, \ldots, p+1$. For the (M)ML formula, Corollary 2.2.1 gives $p=\tilde{p}$ and we find

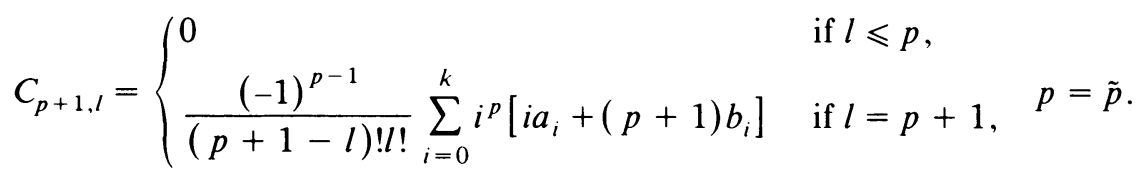

In Table 2.2.1 the numerical values of the constants $C_{p+1, l}$ are explicitly given for two popular choices of the coefficients $\left\{a_{i}, b_{i}\right\}$, viz., the BD formulas and the AM formulas.

For $\theta=0$, and in the case of the ILM formula, Corollary 2.2.1 gives $p=k$, while the coefficients $\left\{b_{i}\right\}$ can still be chosen freely. For the error constants we find

$$
B_{p+1, l}=\frac{(-1)^{k} k !}{(p+1-l) ! l !} \sum_{i=0}^{k} b_{i}, \quad 1 \leqslant l \leqslant p+1, p=k .
$$

For the MML formula, Corollary 2.2.1 gives $p=\tilde{p}$, and for the error constants we find

$$
B_{p+1, l}=\frac{(-1)^{p+1-l}}{(p+1-l) ! l !} \sum_{i=0}^{k} i^{p}\left[i a_{i}+(p+1) b_{i}\right], \quad 1 \leqslant l \leqslant p+1
$$


TABLE 2.2 .1

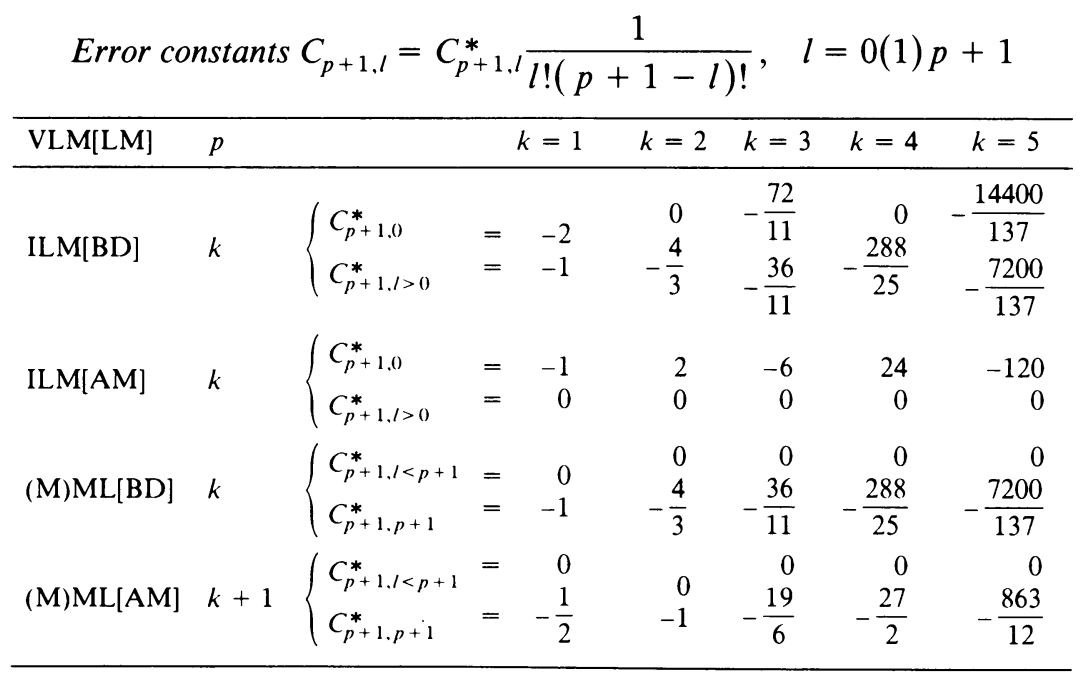

\section{TABLE 2.2 .2}

\begin{tabular}{|c|c|c|c|c|c|c|c|c|}
\hline \multicolumn{9}{|c|}{ Error constants $B_{p+1, l}=B_{p+1, l}^{*} \frac{1}{l !(p+1-l) !}, l=1(1) p+1$} \\
\hline VLM[LM] & $p$ & & & $k=1$ & $k=2$ & $k=3$ & $k=4$ & $k=5$ \\
\hline \multirow{2}{*}{ ILM[BD] } & \multirow{2}{*}{$k$} & \multirow{2}{*}{$B_{p+1.1}^{*}$} & \multirow{2}{*}{$=$} & \multirow{2}{*}{-1} & $\underline{4}$ & 36 & 288 & 7200 \\
\hline & & & & & $\overline{3}$ & $-\overline{11}$ & $\overline{25}$ & 137 \\
\hline \multirow{2}{*}{$\mathrm{MML}[\mathrm{BD}]$} & \multirow{2}{*}{$k$} & \multirow{2}{*}{$(-1)^{p+1-1} B_{p+1.1}^{*}$} & \multirow{2}{*}{$=$} & \multirow{2}{*}{-1} & $\underline{4}$ & 36 & 288 & 7200 \\
\hline & & & & & $\overline{3}$ & $\overline{11}$ & $\overline{25}$ & $\overline{137}$ \\
\hline
\end{tabular}

In Table 2.2.2 the $B_{p+1, l}$ are given for the BD formulas (cf. Table 2.2.1).

2.3. Convergence. We first give a definition of convergence.

Definition 2.3.1. A VLM method is said to yield a convergent solution for (1.1) if $y_{n} \rightarrow y\left(t_{n}\right)$ as $h \rightarrow 0$, with $t_{n}=t$ fixed, holds for all convergent starting values $\left\{y_{i}, Y_{i}\left(t_{j}\right)\right\}, i=1, \ldots, k-1, j=-i,-i+1, \ldots, k-i$.

Before considering the convergence of VLM methods for (1.1), we answer the question as to what equation the numerical scheme (2.1.4) converges to, if we substitute a sufficiently differentiable function $y(t)$ (not necessarily the exact solution) and if we then let $h$ tend to zero in a fixed point. To that end, we define the polynomial

$$
\alpha(z):=\sum_{i=0}^{k} \alpha_{i} z^{k-i}
$$

and the quantities

$$
A_{q}:=\sum_{i=0}^{k}(-i)^{q} \alpha_{i}, \quad q=0,1, \ldots
$$

We observe that $A_{0}=\alpha(1), A_{1}=\alpha^{\prime}(1)-k \alpha(1), \ldots$ 
THEOREM 2.3.1. If sufficiently differentiable functions $y(t), g(t)$ and $K(t, \tau, y)$ are substituted into the VLM method, then the method converges to the equation

$$
\begin{aligned}
& \sum_{q=0}^{m} h^{q}\left\{\frac{\theta A_{q}}{q !} \frac{d^{q}}{d t^{q}} y(t)+\sum_{l=0}^{q}\left[C_{q l}-\frac{A_{q}}{l !(q-l) !}\right]\left(\frac{\partial}{\partial t}\right)^{q-l}\left(\frac{\partial}{\partial s}\right)^{l} Y(t, t)\right\} \\
& =\mathcal{O}\left(h^{r}+h^{m+1}\right) \text { as } h \rightarrow 0,
\end{aligned}
$$

where $r$ is defined in (2.1.3), $C_{q l}$ in (2.2.3), and $m$ is some integer $\geqslant 0$ determined by the differentiability of $y, g$ and $K$.

The proof of Thoerem 2.3.1 is given in Appendix IV.

Examples. In the case of the DQ method for $\{(1.1), \theta=1\}$ we have $A_{0}=1$, so that we infer from Corollary 2.2.1 and Theorem 2.3.1 that the numerical scheme converges to the equation $y(t)-Y(t, t)=0$ as $h \rightarrow 0$, which is the original equation (1.1). In the case of the DQ method for $\{(1.1), \theta=0\}$ we have $C_{00}=1$, so that it easily follows that the numerical scheme converges as $h \rightarrow 0$ to the equation $Y(t, t)=0$, also the original equation (1.1). In the case of the ILM method for (1.1) it is not difficult to show that if the coefficients $\left\{a_{i}, b_{i}\right\}$ in (2.1.4c) correspond to a convergent LM formula for ODEs, then the numerical scheme converges, as $h \rightarrow 0$, to the differentiated Volterra equation $\theta y^{\prime}(t)=K(t, t, y(t))+Y_{t}(t, t)$.

In order to present convergence theorems for VLM methods, we need the following concepts and definitions: A polynomial is called simple von Neumann if its zeros lie on the unit disk, those on the unit circle being simple. A polynomial is called Schur if its zeros lie within the unit circle. Besides $\alpha(z)$ defined above, we define

$$
\begin{array}{ll}
\beta(z):=\sum_{i=0}^{k} \beta_{i} z^{k-i} & \text { where } \beta_{i}:=\sum_{j=-i}^{k-i} \beta_{i j} \\
\gamma(z):=\sum_{i=0}^{k} \gamma_{i} z^{k-i} & \text { where } \gamma_{i}:=\sum_{j=-i}^{k-i} \gamma_{i j} .
\end{array}
$$

Furthermore, we need

$$
\begin{aligned}
& b:=\max _{i, j}\left|\beta_{i j}\right|, \quad c:=\max _{i, j}\left|\gamma_{i j}\right|, \quad w:=\max _{i, j}\left|w_{i j}\right| ; \\
& \left\{\begin{array}{l}
\Delta K\left(t, s, y, y^{*}\right):=K(t, s, y)-K\left(t, s, y^{*}\right), \\
\Delta E(h):=\max _{\substack{i \leqslant j \leqslant N \\
l \leqslant k}}\left|E_{i}\left(h ; t_{j}\right)-E_{i}\left(h ; t_{j+l}\right)\right|,
\end{array}\right. \\
& \left\{\begin{array}{l}
E(h):=\max _{\substack{i \leqslant j \leqslant N \\
l \leqslant k}}\left|E_{i}\left(h ; t_{j+l}\right)\right|, \\
T(h):=\max _{i \leqslant N}\left|\mathbf{L}_{i}(Y)\right|, \\
\delta(h):=\max _{1 \leqslant j \leqslant k^{*}-1}\left|y\left(t_{j}\right)-y_{j}\right|,
\end{array}\right.
\end{aligned}
$$

where $y(t)$ is the exact solution of (1.1), resp. (1.2), and $Y(t, s), Y_{n}(t)$ are the corresponding functions defined in (1.5), resp. (2.1.2). $E(h)$ is the maximal error arising in the approximation of the lag terms $Y_{n}(t)$ during the integration process up 
to $t=T ; T(h)$ may be considered as the maximal local truncation error of the VLM formula (2.1.4) up to $t=T$, and $\delta(h)$ is the maximal starting error.

2.3.1. Second-kind equations. We are now in a position to state a general convergence theorem for VLM methods in the case of second-kind equations $(\theta=1)$, which provides an estimate for the global error

$$
\varepsilon_{n}:=y\left(t_{n}\right)-y_{n} .
$$

THEOREM 2.3.2. Let the conditions for the existence of a unique solution $y \in C(I)$ of $\{(1.1), \theta=1\}$ be satisfied (see Appendix I).

(a) If $\alpha(z) \equiv \alpha_{0} z^{k}, \alpha_{0} \neq 0$, then there exists a constant $C>0$, independent of $h$, such that for $h$ sufficiently small

$$
\left|\varepsilon_{n}\right| \leqslant C[h \delta(h)+E(h)+T(h)], \quad n=k^{*}, \ldots, N .
$$

(b) If $\alpha(z)$ is simple von Neumann, if $\beta(z) \equiv 0$, and if $\Delta K$ satisfies the (uniform) Lipschitz condition

$$
\left|\Delta K\left(t, s, y, y^{*}\right)-\Delta K\left(t^{*}, s, y, y^{*}\right)\right| \leqslant L\left|t-t^{*}\right|\left|y-y^{*}\right|,
$$

for all $(t, s, y),\left(t, s, y^{*}\right),\left(t^{*}, s, y\right),\left(t^{*}, s, y^{*}\right) \in S \times\{|y|<\infty\}$, where the Lipschitz constant $L$ is independent of $t, t^{*}, s, s^{*}, y$ and $y^{*}$, then there exists a constant $C>0$, independent of $h$, such that for $h$ sufficiently small

$$
\left|\varepsilon_{n}\right| \leqslant C h^{-1}[h \delta(h)+\Delta E(h)+T(h)], \quad n=k^{*}, \ldots, N .
$$

The proof of Theorem 2.3.2 is given in Appendix IV.

Using Theorem 2.3.2 it is easy to derive the orders of convergence of the families introduced in Section 2.1.4. The results are given in the following

COROLlaRY 2.3.1. Let $\tilde{p}$ be the order of the LM formula for ODEs defining the coefficients $\left\{a_{i}, b_{i}\right\}$ employed in the ILM and $(M) M L$ methods; let $\delta(h)=\mathcal{O}\left(h^{s}\right)$, $E(h)=\mathcal{O}\left(h^{r}\right)$ and $\Delta E(h)=\mathcal{O}\left(h^{r+1}\right)$ as $h \rightarrow 0$. If the functions $g$ and $K$ are sufficiently smooth, then the order of convergence $p$ is given by

$$
p= \begin{cases}\min \{s+1, r\} & \text { for the } D Q \text { method, } \\ \min \{s, r, \tilde{p}, k\} & \text { for the ILM method, } \\ \min \{s+1, r, \tilde{p}+1\} & \text { for the ML method, } \\ \min \{s, r, \tilde{p}\} & \text { for the MML method }\end{cases}
$$

The convergence analysis of the DQ methods goes back to Kobayasi [10], Linz [12] and Noble [17]. The (M)ML methods were proved to be convergent in Wolkenfelt [22].

It is known (cf. [9], [22]) that VLM methods which have $\beta(z) \equiv 0$ are more stable than DQ methods if large Lipschitz constants for $\partial K / \partial y$ are involved. The maximal attainable order of convergence of these VLM methods is expressed in the following

Corollary 2.3.2. Let $\delta(h)=\mathcal{O}\left(h^{s}\right), \Delta E(h)=\mathcal{O}\left(h^{r+1}\right)$ as $h \rightarrow 0$; let $\alpha(z)$ be simple von Neumann and let $\beta(z) \equiv 0$. Then the order of convergence $p$ of the $k$-step $V L M$ method $\{(2.1 .4) ;(2.1 .2)\}$ satisfies

$$
p \leqslant \begin{cases}\min (s, r, k+1) & \text { for } k \text { odd }, \\ \min (s, r, k+2) & \text { for } k \text { even. }\end{cases}
$$

From this corollary it follows that the MML methods are of maximal attainable order of convergence if we choose the generating $\operatorname{LM}$ formula $\{\rho, \sigma\}$ to be optimal, that is, of order $k+1$ when $k$ is odd, and of order $k+2$ when $k$ is even. We note 
that the restriction $p \leqslant k$ in the ILM methods is due to the use of a $k$-step forward differentiation formula $\left\{\delta_{i}\right\}$ in the generating matrix $B$ (see (2.1.8)).

2.3.2. First-kind equations. Now we shall give convergence theorems for VLM methods for Volterra first-kind equations $(\theta=0)$. We restrict our attention here to linear equations, i.e., we assume in (1.1) that

$$
K(t, \tau, y(\tau))=K(t, \tau) y(\tau) .
$$

We first give the following convergence theorem of Wolkenfelt [20] for $(\rho, \sigma)$-reducible DQ methods.

THEOREM 2.3.3. Let the conditions for the existence of a unique solution $y(t) \in C(I)$ of $\{(1.1), \theta=0\}$ be satisfied (see Appendix I), where $K(t, \tau, y)$ is of the form given in (2.3.8). Let $A=0, B=1, C=0$ in (2.1.7) ( $D Q$ formula) and let the weights in (2.1.2) be given by a $(\rho, \sigma)$-reducible quadrature formula of order $r \geqslant 1$, where $\sigma$ is simple von Neumann. Then there exists a constant $C>0$, independent of $h$, such that for $h$ sufficiently small

$$
\left|\varepsilon_{n}\right| \leqslant C[\delta(h)+E(h)], \quad n=k, \ldots, N .
$$

Proof. See [20].

THEOREM 2.3.4. Let the conditions for the existence of a unique solution $y \in C(I)$ of $\{(1.1), \theta=0\}$ be satisfied. Let $\beta(z) \equiv 0$ and let $\gamma(z)$ be Schur. Then there exists $a$ constant $C>0$, independent of $h$, such that for $h$ sufficiently small

$$
\left|\varepsilon_{n}\right| \leqslant C h^{-1}[h \delta(h)+\Delta E(h)+T(h)], \quad n=k^{*}, \ldots, N .
$$

The proof of Theorem 2.3.4 is given in Appendix IV.

Observe that this convergence result is identical to that obtained for VLM methods for $\{(1.1), \theta=1\}$ with $\beta(z) \equiv 0$ (Theorem 2.3.2(b)). Now it is easy to derive from Theorems 2.3.3 and 2.3.4 the orders of convergence of the DQ, ILM and MML methods for $\{(1.1), \theta=0\}$. The results are summarized in the following

COROLlary 2.3.3. Let $\bar{p}$ be the order of the LM formula $\{\rho, \sigma\}$ employed in the $D Q$ lag term formula (2.1.2) and let $\tilde{p}$ be the order of the LM formula for ODEs employed in the MML formula (2.1.10) (with $A \equiv 0$ ). Furthermore, let $\delta(h)=\mathcal{O}\left(h^{s}\right), E(h)=$ $\mathcal{O}\left(h^{r}\right)$ and $\Delta E(h)=\mathcal{O}\left(h^{r+1}\right)$ as $h \rightarrow 0$. If the functions $g$ and $K$ are sufficiently smooth, then the order of convergence $p$ is given by

$$
p= \begin{cases}\min \{s, \bar{p}\} & \text { for the DQ method with } \sigma \text { being simple von Neumann, } \\ \min \{s, r, k\} & \text { for the ILM method with } \gamma \text { being Schur } \\ \min \{s, r, \tilde{p}\} & \text { for the MML method with } \gamma \text { being Schur. }\end{cases}
$$

Wolkenfelt [21] has also given a convergence theorem for MML methods for nonlinear equations $\{(1.1), \theta=0\}$, with the following restrictions on the coefficients $\beta_{i j}$ and $\gamma_{i j}: \beta(z) \equiv 0, \gamma_{00} \neq 0$ and all other $\gamma_{i j}$ vanish.

2.4. Numerical Experiments. In this section we present the results of numerical experiments in order to support and illustrate the convergence behavior of VLM methods for (1.1), as predicted by Corollaries 2.3.1 and 2.3.3, by applying various DQ, ILM and (M)ML methods to a number of problems. In addition, for second-kind equations we illustrate the behavior of the various methods when the kernel $K(t, \tau, y)$ has a large Lipschitz constant with respect to $y$. 
The required starting values for $y_{i}, 0 \leqslant i<n_{1}+k$, are taken from the exact solution $y\left(t_{i}\right)$ (so that $s=\infty$ in Corollaries 2.3.1 and 2.3.3), and values of the lag term $Y_{n}(t)$ required in (2.1.4) for $n \geqslant n_{1}$ are computed with a Gregory quadrature rule in (2.1.2) of the proper order. The coefficients $\left\{a_{i}, b_{i}\right\}$ in the ILM and (M)ML formulas are taken from Tables 2 and 3 of Appendix II. The values of $r, \tilde{p}$ and $k$ in Corollary 2.3.1 and $r, \bar{p}, \tilde{p}$ and $k$ in Corollary 2.3.3 are chosen as small as is allowed by the theoretical order to be tested.

In the tables of results, we present the number of correct significant digits at the endpoint $T$, i.e., the value of

$$
\operatorname{sd}(h):=-\log _{10}\left(\left|y(T)-y_{N}\right| /|y(T)|\right), \quad T=t_{N}=N h .
$$

Moreover, we list the effective order of the method, viz., the value of

$$
(\operatorname{sd}(h)-\operatorname{sd}(2 h)) / \log _{10} 2 \text {. }
$$

This value should tend to the asymptotic order of convergence as $h \rightarrow 0$ and will tell us therefore

(i) whether the asymptotic, theoretical order of the numerical scheme is correct, and

(ii) how fast the asymptotic order is reached.

2.4.1. Second-kind equations $((1.1)$ with $\theta=1)$.

Example 2.4.1 (Garey [5], adapted).

$$
\begin{aligned}
K(t, \tau, y) & =-\lambda \cdot \ln (1+t-\tau) y, \\
g(t) & =1-t+\lambda\left[\frac{1}{2}\left(1-t^{2}\right) \ln (1+t)+\frac{3}{4} t^{2}-\frac{1}{2} t\right], \\
y(t) & =1-t, \quad\left[t_{0}, T\right]=[0,4] .
\end{aligned}
$$

For $\lambda=4$, Table 2.4 .1 gives the results obtained with DQ, ILM and (M)ML methods of asymptotic order 5 , where for the coefficients $\left\{a_{i}, b_{i}\right\}$ employed in the ILM and (M)ML methods we used the coefficients of the Adams-Moulton formula of the proper order. $G_{r}$ means that for the lag term we used a Gregory rule of order $r$ and $\mathrm{AM}_{\tilde{p}}$ means that a $\tilde{p}$ th order Adams-Moulton formula was used.

The example with $\lambda=4$ shows that the correct asymptotic order $p=5$ is already reached by all methods for not too small integration steps. For relatively large values of $h$ the MML method shows the most accurate results. The ILM method shows an accuracy about 1-2 digits less than the other methods, because of larger error constants (cf. Table 2.2.1).

TABLE 2.4.1

Example 2.4.1 with $\lambda=4$

\begin{tabular}{ccccc}
\hline$h$ & $\mathrm{DQ}\left(\mathrm{G}_{5}\right)$ & $\mathrm{ILM}\left(\mathrm{G}_{5}-\mathrm{AM}_{6}\right)$ & $\mathrm{ML}\left(\mathrm{G}_{5}-\mathrm{AM}_{4}\right)$ & $\mathrm{MML}\left(\mathrm{G}_{5}-\mathrm{AM}_{5}\right)$ \\
\hline $1 / 4$ & 4.6 & 3.4 & 4.3 & 6.1 \\
& $>4.6$ & $>3.8$ & $>4.8$ & $>3.9$ \\
$1 / 8$ & 6.0 & 4.5 & 5.7 & 7.3 \\
& $>5.0$ & $>4.5$ & 7.1 & $>4.6$ \\
$1 / 16$ & 7.5 & 5.9 & $>5.0$ & $>3.2$ \\
& $>5.0$ & $>4.7$ & 8.6 & 9.4 \\
$1 / 32$ & 9.0 & 7.3 & $>5.0$ & $>4.6$ \\
$1 / 64$ & 10.5 & 8.8 & 10.1 & 10.8 \\
\hline
\end{tabular}


TABLE 2.4.2

Example 2.4.1 with $\lambda=100$ ( $*$ indicates instability)

\begin{tabular}{lccccccc}
\hline & & \multicolumn{2}{c}{$\mathrm{ILM}$} & \multicolumn{2}{c}{$\mathrm{ML}$} & \multicolumn{2}{c}{$\mathrm{MML}$} \\
\multicolumn{1}{c}{$\mathrm{DQ}\left(\mathrm{G}_{5}\right)$} & $\left(\mathrm{G}_{5}-\mathrm{AM}_{6}\right)\left(\mathrm{G}_{5}-\mathrm{BD}_{5}\right)$ & $\left(\mathrm{G}_{5}-\mathrm{AM}_{4}\right)\left(\mathrm{G}_{5}-\mathrm{BD}_{4}\right)$ & $\left(\mathrm{G}_{5}-\mathrm{AM}_{5}\right)\left(\mathrm{G}_{5}-\mathrm{BD}_{5}\right)$ \\
\hline $1 / 4$ & -6.5 & 1.8 & 3.1 & $*$ & $*$ & $*$ & $*$ \\
$1 / 8$ & 2.3 & 4.5 & 3.6 & 3.7 & $*$ & 4.2 & $*$ \\
$1 / 16$ & 6.3 & 5.8 & 5.8 & 6.2 & 2.2 & 9.0 & 5.6 \\
\hline
\end{tabular}

TABLE 2.4 .3

Example 2.4.2

\begin{tabular}{|c|c|c|c|c|}
\hline$h$ & $\mathrm{DQ}\left(\mathrm{G}_{1}\right.$ & $\operatorname{ILM}\left(\mathrm{G}_{5}-\mathrm{BD}_{5}\right)$ & $\mathrm{ML}\left(\mathrm{G}_{5}-\mathrm{BD}_{4}\right)$ & $\operatorname{MML}\left(\mathrm{G}_{5}-\mathrm{BD}_{5}\right)$ \\
\hline $1 / 2$ & $*(2.5)$ & $\begin{array}{l}1.57 \\
>7.7\end{array}$ & $*(4.5)$ & $*(5.0)$ \\
\hline $1 / 4$ & $*(2.25)$ & $\begin{array}{l}3.88 \\
>7.4\end{array}$ & $*(3.25)$ & $*(4.0)$ \\
\hline $1 / 8$ & $*(3.375)$ & $\begin{array}{l}6.11 \\
\quad>5.2\end{array}$ & $*(2.875)$ & $*(3.625)$ \\
\hline $1 / 16$ & $*(4.6875)$ & 7.68 & $*(3.5625)$ & $*(5.4375)$ \\
\hline
\end{tabular}

The example with $\lambda=100$ shows that the ILM method is stable for "realistic" values of $h$ (in view of the behavior of the exact solution, integration steps $h=1 / 4$ or $h=1 / 8$ should be small enough for representing the function $y(t)=1-t$ ), whereas the other methods develop instabilities. Comparing the results obtained for $\lambda=4$ and $\lambda=100$ we see that the ILM method is insensitive to larger values of $\lambda$ already for $h \leqslant 1 / 8$, whereas the other methods become insensitive only for much smaller values of $h$.

Example 2.4.2. As a further test of the behavior of VLM methods for stiff problems we have integrated the nonlinear Hammerstein-type equation defined by

$$
\begin{aligned}
& K(t, \tau, y)=-[16(t-\tau)+1] e^{y}, \\
& g(t)=-15 t+17\left(e^{t}-1\right), \\
& y(t)=t, \quad\left[t_{0}, T\right]=[0,8] .
\end{aligned}
$$

The nonlinear equations which emerge when we apply a VLM method to this problem, are solved with Newton's process. The Newton iteration is stopped as soon as $\left|y_{n}^{(i+1)}-y_{n}^{(i)}\right| \leqslant 10^{-12}\left(1+\left|y_{n}^{(i+1)}\right|\right)$, where $y_{n}^{(i)}$ is the $i$ th Newton approximation to $y_{n}$, with the restriction that not more than 10 Newton iterations are allowed. Table 2.4.3 presents the results. An " $*(t)$ " indicates that at a point $t$ the Newton iteration process had not yet converged after 10 steps, and in all these cases the numerical process clearly showed an unstable behavior. The results show the superiority of the ILM method for this stiff problem. In [8] more experiments are given with the ILM method, together with a comparison with a block-implicit Runge-Kutta method of de Hoog and Weiss. 
TABLE 2.4.4

Example 2.4.3 with $\varepsilon=10^{-4}$ and $\varepsilon=10^{-6}(*$ indicates instability $)$

\begin{tabular}{lcccccccc}
\hline$h$ & \multicolumn{2}{c}{$\mathrm{DQ}\left(\mathrm{G}_{5}\right)$} & \multicolumn{2}{c}{$\mathrm{ILM}\left(\mathrm{G}_{5}-\mathrm{AM}_{6}\right)$} & \multicolumn{2}{c}{$\mathrm{ML}\left(\mathrm{G}_{5}-\mathrm{AM}_{4}\right)$} & \multicolumn{2}{c}{$\mathrm{MML}\left(\mathrm{G}_{5}-\mathrm{AM}_{5}\right)$} \\
& $\varepsilon=10^{-4}$ & $\varepsilon=10^{-6}$ & $\varepsilon=10^{-4}$ & $\varepsilon=10^{-6}$ & $\varepsilon=10^{-4}$ & $\varepsilon=10^{-6}$ & $\varepsilon_{\varepsilon=10^{-4}}$ & $\varepsilon=10^{-6}$ \\
\hline $1 / 4$ & .20 & .02 & $*$ & $*$ & .17 & .02 & 5.4 & 6.6 \\
$1 / 8$ & .37 & .05 & $*$ & $*$ & .34 & .04 & 5.0 & 6.4 \\
$1 / 16$ & .90 & .09 & $*$ & $*$ & .58 & .08 & 4.8 & 6.3 \\
\hline
\end{tabular}

Example 2.4.3 (Weakly singular equations). Finally we show that VLM methods may be used for integrating weakly singular equations, a standard example of which is given by [19]

$$
y(t)=1+2 \sqrt{t}-\int_{0}^{t} \frac{y(\tau) d \tau}{\sqrt{t-\tau}}, \quad 0 \leqslant t \leqslant 4,
$$

with the exact solution $y(t)=1$. We cannot directly apply a VLM method to this equation; however, by approximating the square root $\sqrt{t-\tau}$ by $\sqrt{t-\tau+\varepsilon}$ with $\varepsilon$ a small, positive parameter, the equation becomes nonsingular and in principle the VLM methods do apply. The numerical solution $\left\{y_{n}(\varepsilon)\right\}_{n=k^{*}}^{N}$ obtained is expected to present an accurate approximation if $h$ and $\varepsilon$ are sufficiently small.

In choosing an appropriate VLM method we should take into account that the kernel $-y / \sqrt{t-\tau+\varepsilon}$ has a derivative $\partial K / \partial y=-1 / \sqrt{t-\tau+\varepsilon}$ which is large negative for $t \approx \tau$. This strongly suggests choosing a VLM formula that does not use kernel values on or close to the line $t=s$, i.e., the matrix $C=\left(\gamma_{i j}\right)$ should have its nonzero entries preferably off the "diagonal" $j=-i$. Among the special families of VLM formulas discussed in Section 2.1.4, the ML and MML formulas (2.1.9) and (2.1.10) are formulas having just one nonzero entry $\gamma_{0,0}$ on the "diagonal" $j=-i$. It has already been observed in Section 2.1.2 that the ML methods may become unstable if large Lipschitz constants are involved. The ILM formulas only use $\gamma_{i j}$ 's on the "diagonal" and therefore they should not be expected to work on our "almost" singular equation.

The above heuristic arguments are completely confirmed by the results presented in Table 2.4.4. Moreover, this experiment indicates that the MML methods are to be considered as promising methods for weakly singular equations of the type given here, and that it would be interesting to compare them with other methods for this type of integral equations (see, e.g., [19]).

2.4.2. First-kind equations $((1.1)$ with $\theta=0)$.

Example 2.4.4 (Gladwin [6]).

$$
\begin{aligned}
K(t, \tau, y) & =\cos (t-\tau) y, \\
g(t) & =-\exp (t)-\sin (t)+\cos (t), \\
y(t) & =\exp (t), \quad\left[t_{0}, T\right]=[0,4] .
\end{aligned}
$$

Table 2.4.5 gives the results obtained with DQ, ILM and MML methods of asymptotic order 4 and 5 , where for the coefficients $\left\{a_{i}, b_{i}\right\}$ in the ILM and MML methods we used the coefficients of the backward differentiation formulas of the proper order. $\mathrm{BD}_{k}$ means that a $k$-step ( $k$ th order) $\mathrm{BD}$ formula was used. Notice that these special ILM methods are identical to (2.1.4b). 
TABLE 2.4 .5

Example 2.4 .4

\begin{tabular}{|c|c|c|c|c|c|c|}
\hline$h$ & $\mathrm{DQ}\left(\mathrm{G}_{4}\right)$ & $\operatorname{ILM}\left(G_{4}-B_{4}\right)$ & $\operatorname{MML}\left(G_{4}-\mathrm{BD}_{4}\right)$ & $\mathrm{DQ}\left(\mathrm{G}_{5}\right)$ & $\operatorname{ILM}\left(G_{5}-B_{5}\right)$ & $\operatorname{MML}\left(G_{5}-B D_{5}\right)$ \\
\hline $1 / 10$ & -7.6 & $\begin{array}{l}4.3 \\
\quad>4.1\end{array}$ & $\begin{array}{l}3.9 \\
\quad>3.8\end{array}$ & -11 & $\begin{array}{l}5.6 \\
\quad>4.9\end{array}$ & $\begin{array}{l}4.9 \\
>4.9\end{array}$ \\
\hline $1 / 20$ & -21 & $\begin{array}{l}5.5 \\
\quad>4.0\end{array}$ & $\begin{array}{l}5.1 \\
\quad>3.9\end{array}$ & -29 & $\begin{array}{l}7.0 \\
\quad>5.0\end{array}$ & $\begin{array}{l}6.4 \\
\quad>5.0\end{array}$ \\
\hline $1 / 40$ & -50 & $\begin{array}{l}6.7 \\
\quad>4.0\end{array}$ & $\begin{array}{l}6.3 \\
\quad>4.0\end{array}$ & -65 & $\begin{array}{l}8.5 \\
> \\
5.2\end{array}$ & $\begin{array}{l}7.9 \\
>5.0\end{array}$ \\
\hline $1 / 80$ & -109 & 7.9 & 7.5 & -140 & 10.1 & 9.4 \\
\hline
\end{tabular}

For the ILM and the MML methods the results show that the correct asymptotic order is reached already for relatively large values of $h$. The apparent unstable behavior of the $\mathrm{DQ}\left(\mathrm{G}_{4}\right)$ and $\mathrm{DQ}\left(\mathrm{G}_{5}\right)$ methods is explained by the fact that the Gregory quadrature formulas of order $\geqslant 3$ are $(\rho, \sigma)$-quadrature formulas for which the $\sigma$-polynomial is not simple von Neumann (cf. Corollary 2.3.3). Unlike its performance for second-kind equations, the ILM method is here more accurate than the MML method.

3. VLM Methods for Volterra Integro-Differential Equations.

3.1. The General VLM Method. In analogy to the VLM formula (2.1.4) for Volterra integral equations we formally define the VLM formula for Volterra integro-differential equations (1.2) as follows:

$$
\begin{array}{r}
\sum_{i=0}^{k} \alpha_{i}^{*} y_{n-i}=h \sum_{i=0}^{k} \gamma_{i}^{*} f_{n-i}, \quad f_{n}:=f\left(t_{n}, y_{n}, z_{n}\right), \\
\sum_{i=0}^{k} \alpha_{i} z_{n-i}+\sum_{i=0}^{k} \sum_{j=-k}^{k} \beta_{i j} Y_{n-i}\left(t_{n+j}\right)=h \sum_{i=0}^{k} \sum_{j=-k}^{k} \gamma_{i j} K_{n-i}\left(t_{n+j}\right), \\
n=k^{*}(1) N,
\end{array}
$$

where $Y_{n}(t)$ is defined as in (2.1.2) and $\left\{\alpha_{i}^{*}, \gamma_{i}^{*}\right\}_{i=0}^{k}$ are the coefficients of some LM method for ODEs. These formulas (3.1.1), combined with (2.1.2), will be called a $V L M$ method for integro-differential equations. Formula $(3.1 .1 \mathrm{~b})$ can be characterized by the parameter matrices $A=\left(\alpha_{i}\right), B=\left(\beta_{i j}\right), C=\left(\gamma_{i j}\right)$.

When we compare (3.1.1) with (2.1.4), it is clear that all methods defined for second-kind Volterra integral equations, by specifying the matrices $A, B$ and $C$ in (2.1.7) and the quadrature weights $w_{n j}$ in (2.1.2), can be extended to methods for Volterra integro-differential equations by specifying the coefficients $\left\{\alpha_{i}^{*}, \beta_{i}^{*}\right\}$ of some LM method for ODEs. In this way, we define DQ, ILM, ML and MML methods for (1.2), where the matrices $A, B$ and $C$ are specified in Section 2.1.4 for the families $1,2,3$ and 4 , respectively. For example, any DQ method for (1.2) is specified by $A=I, B=-I, C=0$, which gives $z_{n}=Y_{n}\left(t_{n}\right)$ for (3.1.1b).

An alternative way to arrive at the VLM formulas (3.1.1) is obtained as follows. We first integrate (1.2) formally, which results in the system of Volterra integral 
equations of the second kind

$$
\left\{\begin{array}{l}
y(t)=y\left(t_{0}\right)+\int_{t_{0}}^{t} f(\tau, y(\tau), z(\tau)) d \tau, \\
z(t)=g(t)+\int_{t_{0}}^{t} K(t, \tau, y(\tau)) d \tau
\end{array}\right.
$$

Next, we apply the VLM formula (2.1.4) to this system with parameter matrices $\left(A^{*}, B^{*}, C^{*}\right)$ and $(A, B, C)$ for the respective components, i.e.,

$$
\begin{aligned}
& \sum_{i=0}^{k} \alpha_{i}^{*} y_{n-i}+\sum_{i=0}^{k} \sum_{j=-k}^{k} \beta_{i j}^{*} Y_{n-i}^{*}\left(t_{n+j}\right)=h \sum_{i=0}^{k} \sum_{j=-k}^{k} \gamma_{i j}^{*} f\left(t_{n-i}, y_{n-i}, z_{n-i}\right), \\
& \sum_{i=0}^{k} \alpha_{i} z_{n-i}+\sum_{i=0}^{k} \sum_{j=-k}^{k} \beta_{i j} Y_{n-i}\left(t_{n+j}\right)=h \sum_{i=0}^{k} \sum_{j=-k}^{k} \gamma_{i j} K\left(t_{n+j}, t_{n-i}, y_{n-i}\right),
\end{aligned}
$$

where $Y_{n}^{*}\left(t_{j}\right)$ is an approximation to

$$
Y^{*}(t, s):=y\left(t_{0}\right)+\int_{t_{0}}^{s} f(\tau, y(\tau), z(\tau)) d \tau
$$

at $t=t_{j}, s=t_{n}$. Here, however, both $Y^{*}$ and $f$ do not depend on $t$ so that, by putting $\beta_{i}^{*}:=\sum_{j=-k}^{k} \beta_{i j}^{*}=0$ and writing $\gamma_{i}^{*}:=\sum_{j=-k}^{k} \gamma_{i j}^{*}$, we have reduced (3.1.1') to (3.1.1).

3.2. Consistency of VLM Formulas for Integro-Differential Equations. With the numerical schemes (3.1.1) we associate the linear difference-differential operators $\mathbf{L}_{n}^{*}: C^{1}(I) \rightarrow \mathbf{R}$ and $\mathbf{L}_{n}: C_{2}^{1}(S) \rightarrow \mathbf{R}$, defined by

$$
\mathbf{L}_{n}^{*}[y]:=\sum_{i=0}^{k}\left[\alpha_{i}^{*}-\gamma_{i}^{*} h \frac{d}{d t}\right] y\left(t_{n-i}\right)
$$

and

$$
\mathbf{L}_{n}[Y]:=\sum_{i=0}^{k}\left\{\alpha_{i} Y\left(t_{n-i}, t_{n-i}\right)+\sum_{j=-k}^{k}\left[\beta_{i j}-\gamma_{i j} h \frac{\partial}{\partial s}\right] Y\left(t_{n+j}, t_{n-i}\right)\right\}
$$

where $y$ and $Y$ are arbitrary functions from $C^{1}(I)$ and $C_{2}^{1}(S)$, respectively. Now we substitute the exact solution $y(t)$ and $z(t)$ of (1.2) into (3.2.1) and obtain (cf. (2.2.2))

$$
\begin{gathered}
\sum_{i=0}^{k}\left[\alpha_{i}^{*} y\left(t_{n-i}\right)-\gamma_{i}^{*} h f\left(t_{n-i}, y\left(t_{n-i}\right), z\left(t_{n-i}\right)\right)\right]=\mathbf{L}_{n}^{*}[y], \\
\sum_{i=0}^{k}\left\{\alpha_{i} z\left(t_{n-i}\right)+\sum_{j=-k}^{k}\left[\beta_{i j} \tilde{Y}_{n-i}\left(t_{n+j}\right)-\gamma_{i j} h \tilde{K}_{n-i}\left(t_{n+j}\right)\right]\right\} \\
=\mathbf{L}_{n}[Y]-\sum_{i=0}^{k} \sum_{j=-k}^{k} \beta_{i j} E_{n-i}\left(h ; t_{n+j}\right) \\
=\mathbf{L}_{n}[Y]+\mathcal{O}\left(h^{r}\right) \text { as } h \rightarrow 0,
\end{gathered}
$$

where $\tilde{Y}_{n-i}\left(t_{n+j}\right)$ and $\tilde{K}_{n-i}\left(t_{n+j}\right)$ are defined by (2.1.2) and (2.1.1) with $y_{n}$ replaced by $y\left(t_{n}\right)$, and where $r$ is the order of the quadrature error $E_{n}$. This shows the connection of the operators (3.2.1) with the VLM formula (3.1.1). The quantities $\mathbf{L}_{n}^{*}[y]$ and $\mathbf{L}_{n}[Y]$, with $y$ and $Y$ corresponding to the exact solution of (1.2), are 
called the local truncation errors of the VLM formulas (3.1.1). In analogy to Section 2.2 we use the following

Definition 3.2.1. The operators (3.2.1) and the associated VLM formulas (3.1.1) are said to be of order $p^{*}$ and $p$ if for all $y \in C^{p^{*+1}}(I)$ and for all $Y \in C^{p+1}(S)$, we have $\mathbf{L}^{*}[y]=\mathcal{O}\left(h^{p^{*}+1}\right)$ and $\mathbf{L}_{n}[Y]=\mathcal{O}\left(h^{p+1}\right)$ as $h \rightarrow 0$, with nonvanishing error constants.

Since $\mathbf{L}_{n}^{*}$ is of the same form as the linear operator $\mathbf{L}_{n}$ occurring in ODE theory (compare Lambert [11, p. 23]) the consistency conditions for $\mathbf{L}_{n}^{*}$ are also of the same form. (It should be remarked that in the derivation of the order conditions we expand $y\left(t_{n-i}\right)$ and $y^{\prime}\left(t_{n-i}\right)$ as Taylor series about $t_{n}$, whereas Lambert expands about $t_{n-k}$.) Similarly, since $\mathbf{L}_{n}$ defined in (3.2.1b) is identical to the operator defined in (2.2.1), the order conditions for $\mathbf{L}_{n}$ are also known already. Therefore, the following theorem is immediate.

THEOREM 3.2.1. The operators $\mathbf{L}_{n}^{*}$ and $\mathbf{L}_{n}$ and the associated VLM formulas (3.1.1) are of order $p^{*}$ and $p$, if $C_{q}=0$ for $q=0,1, \ldots, p^{*}$ and $C_{q l}=0$ for $q=0,1, \ldots, p$, $l=0,1, \ldots, q$, where

$$
C_{q}:=\frac{(-1)^{q}}{q !} \sum_{i=0}^{k} i^{q-1}\left[i \alpha_{i}^{*}+q \gamma_{i}^{*}\right]
$$

and where $C_{q l}$ is defined in (2.2.3).

Evidently, $p^{*}$ equals the order of the LM method for ODEs with coefficients $\left\{\alpha_{i}^{*}, \gamma_{i}^{*}\right\}$. Furthermore, in the case of the DQ, ILM, ML and MML formulas, $p$ is determined by the expressions as derived for the operator $\mathbf{L}_{n}$ for second-kind Volterra equations in Corollary 2.2.1.

The values of the error constants $C_{p^{*}+1}$ and $C_{p+1, l}, 0 \leqslant l \leqslant p+1$, follow easily from those given in Table 2.2.1 (for a number of popular methods for second-kind Volterra integral equations).

3.3. Convergence. As we did for the first- and second-kind Volterra equations, we first consider the continuous problem to which the VLM method $\{(3.1 .1) ;(2.1 .2)\}$ converges as $h \rightarrow 0$. We assume that the LM formulas in (3.1.1) are consistent and that $A_{1}=\alpha^{\prime}(1)-k \alpha(1) \neq 0$ (see Section 2.3). Then, for sufficiently smooth functions $g, K$ and $f$, the VLM method converges to the equations

$$
\begin{aligned}
& y^{\prime}(t)=f(t, y(t), z(t)), \\
& \alpha(1)[z(t)-Y(t, t)]+\left(\alpha^{\prime}(1)-k \alpha(1)\right) h\left[z^{\prime}(t)-Y_{t}(t, t)-Y_{s}(t, t)\right]=0
\end{aligned}
$$

as $h \rightarrow 0$ (see the proof of Theorem 2.3.1). Thus, if $\alpha(1) \neq 0$ (DQ and ML method), then the VLM method is a direct discretization of (1.2). If $\alpha(1)=0$ (ILM and MML) (and $\alpha^{\prime}(1) \neq 0$ by assumption), then the linear method converges to the system

$$
\begin{aligned}
y^{\prime}(t) & =f(t, y(t), z(t)) \\
z^{\prime}(t) & =K(t, t, y(t))+g^{\prime}(t)+\int_{t_{0}}^{t} K_{t}(t, \tau, y(\tau)) d \tau,
\end{aligned}
$$

that is, to the system (1.2) where the expression for $z(t)$ is differentiated with respect to $t$. 
Next, we present a general convergence theorem. In the proof it is convenient to use, in addition to the notation introduced in Section 2.3, the notations

$$
\begin{aligned}
& \eta_{n}=z\left(t_{n}\right)-z_{n}, \\
& \Delta f_{n}:=f\left(t_{n}, y\left(t_{n}\right), z\left(t_{n}\right)\right)-f\left(t_{n}, y_{n}, z_{n}\right), \\
& T^{*}(h):=\max _{i \leqslant N}\left|\mathbf{L}_{i}^{*}[y]\right|, \quad \delta^{*}(h):=\max _{j \leqslant k-1}\left|z\left(t_{j}\right)-z_{j}\right|, \\
& \alpha^{*}(z):=\sum_{i=0}^{k} \alpha_{i}^{*} z^{k-i},
\end{aligned}
$$

where $z$ and $y$ correspond to the exact solution.

THEOREM 3.3.1. Let the conditions for the existence of a unique solution $y \in C^{1}(I)$ of (1.2) be satisfied (see Appendix I). Let $\alpha(z)$ and $\alpha^{*}(z)$ be simple von Neumann.

(a) If $\alpha(z)=\alpha_{0} z^{k}, \alpha_{0} \neq 0$, then there exists a constant $C>0$, independent of $h$, such that for $h$ sufficiently small,

$$
\left|\varepsilon_{n}\right| \leqslant C\left[\delta(h)+h \delta^{*}(h)+E(h)+T(h)+h^{-1} T^{*}(h)\right], \quad n=k^{*}, \ldots, N .
$$

(b) If $\beta(z) \equiv 0$, then there exists a constant $C>0$, independent of $h$, such that for $h$ sufficiently small,

$$
\begin{aligned}
\left|\varepsilon_{n}\right| \leqslant C\left[\delta(h)+\delta^{*}(h)+h^{-1} \Delta E(h)+h^{-1} T(h)+h^{-1} T^{*}(h)\right], & \\
n & =k^{*}, \ldots, N .
\end{aligned}
$$

The proof of Theorem 3.3.1 is given in Appendix IV.

Using this theorem, it is easy to derive the orders of convergence of the various examples of VLM methods for Volterra integro-differential equations, described in Section 3.1. The results are given in the following

Corollary 3.3.1. Let $p^{*}$ and $\tilde{p}$ be the orders of the VLM formula $\left\{a_{i}^{*}, \gamma_{i}^{*}\right\}$ employed in (3.1.1a) and of the LM formula $\left\{a_{i}, b_{i}\right\}$ employed in the (M)ML and $I L M$ methods, respectively; let $\delta(h)=\mathcal{O}\left(h^{s}\right), \delta^{*}(h)=\mathcal{O}\left(h^{s^{*}}\right), E(h)=\mathcal{O}\left(h^{r}\right), \Delta E(h)$ $=\mathcal{O}\left(h^{r+1}\right)$ as $h \rightarrow 0$. Let the functions $f, g$ and $K$ be sufficiently smooth. Then, the order of convergence $p$ of the VLM method $\{(3.1 .1) ;(2.1 .2)\}$ is given by

$$
p= \begin{cases}\min \left(s, s^{*}+1, r, p^{*}\right) & \text { for the DQ method, } \\ \min \left(s, s^{*}+1, r, p^{*}, \tilde{p}+1\right) & \text { for the ML method, } \\ \min \left(s, s^{*}, r, p^{*}, \tilde{p}\right) & \text { for the MML method, } \\ \min \left(s, s^{*}, r, p^{*}, \tilde{p}, k\right) & \text { for the ILM method. }\end{cases}
$$

The convergence of the conventional DQ method has already been studied by Linz [13] and Mocarsky [15]. The convergence results for the (M)ML methods have already been given in Wolkenfelt [22].

3.4. Numerical Experiments. In order to illustrate the convergence behavior of VLM methods for (1.2), we have tested the DQ, ILM and (M)ML methods of orders 2,3 and 4. For the two ODE-LM formulas involved in (3.1.1) we choose the backward differentiation formulas. As in the experiments for (1.1), the lag term $Y_{n}(t)$ was evaluated with a Gregory quadrature rule of the proper order. 
EXAMPLE 3.4.1 (Linz [13], Mocarsky [15], Makroglou [14]).

$$
\begin{aligned}
& f(t, y, z)=1-t \cdot \exp \left(-t^{2}\right)+y+2 z, \quad y(0)=0, \\
& K(t, \tau, y)=t \tau \exp \left(-y^{2}\right), \quad g(t)=0, \\
& y(t)=t, \quad\left[t_{0}, T\right]=[0,2] .
\end{aligned}
$$

Table 3.4.1 gives the results of our experiments. The ILM method is the less accurate one, the DQ and (M)ML methods exhibit a varying accuracy behavior.

TABLE 3.4.1

\begin{tabular}{|c|c|c|c|c|}
\hline$h$ & $\mathrm{DQ}\left(\mathrm{G}_{2}\right)$ & $\operatorname{ILM}\left(\mathrm{G}_{2}-\mathrm{BD}_{2}\right)$ & $\mathrm{ML}\left(\mathrm{G}_{2}-\mathrm{BD}_{1}\right)$ & $\operatorname{MML}\left(\mathrm{G}_{2}-\mathrm{BD}_{2}\right)$ \\
\hline \multirow[t]{2}{*}{$1 / 10$} & 2.2 & 3.3 & 2.2 & 1.8 \\
\hline & $>2.0$ & $>-2$ & $>2.2$ & $>2.0$ \\
\hline \multirow[t]{2}{*}{$1 / 20$} & 2.8 & 2.6 & 2.8 & 2.4 \\
\hline & $>2.0$ & $>1.3$ & $>2.0$ & $>2.0$ \\
\hline $1 / 40$ & 3.4 & 3.0 & 3.5 & 3.0 \\
\hline
\end{tabular}

Example 3.4.1

second-order methods (with $\left\{\alpha_{i}^{*}, \gamma_{i}^{*}\right\}=\mathrm{BD}_{2}$ in (3.1.1a))

third-order methods (with $\mathrm{BD}_{3}$ in (3.1.1a))

\begin{tabular}{|c|c|c|c|c|}
\hline$h$ & $\mathrm{DQ}\left(\mathrm{G}_{4}\right)$ & $\operatorname{ILM}\left(G_{4}-B D_{4}\right)$ & $\mathrm{ML}\left(\mathrm{G}_{4}-\mathrm{BD}_{3}\right)$ & $\operatorname{MML}\left(G_{4}-B D_{4}\right)$ \\
\hline \multirow[t]{2}{*}{$1 / 10$} & 4.0 & 3.2 & 3.6 & 3.6 \\
\hline & $>3.7$ & $>4.5$ & $>4.0$ & $>3.1$ \\
\hline \multirow[t]{2}{*}{$1 / 20$} & 5.1 & 4.6 & 4.8 & 4.6 \\
\hline & $>3.9$ & $>6.2$ & $>4.1$ & $>3.9$ \\
\hline $1 / 40$ & 6.3 & 6.4 & 6.1 & 5.7 \\
\hline
\end{tabular}

\begin{tabular}{ccccc}
\hline$h$ & $\mathrm{DQ}\left(\mathrm{G}_{3}\right)$ & $\mathrm{ILM}\left(\mathrm{G}_{3}-\mathrm{BD}_{3}\right)$ & $\mathrm{ML}\left(\mathrm{G}_{3}-\mathrm{BD}_{2}\right)$ & $\mathrm{MML}\left(\mathrm{G}_{3}-\mathrm{BD}_{3}\right)$ \\
\hline $1 / 10$ & 3.6 & 2.4 & 2.9 & 3.3 \\
& $>2.8$ & $>2.3$ & $>2.7$ & $>4.7$ \\
$1 / 20$ & 4.5 & 3.1 & 3.7 & 4.7 \\
$1 / 40$ & 5.4 & 3.9 & 4.6 & 6.0 \\
\hline
\end{tabular}

fourth-order methods (with $\mathrm{BD}_{4}$ in (3.1.1a))

Acknowledgement. We would like to thank Joke Blom for programming the numerical experiments.

Department of Numerical Mathematics

Centre for Mathematics and Computer Science

Kruislaan 413

1098 SJ Amsterdam, The Netherlands

1. C. T. H. Baker, The Numerical Treatment of Integral Equations, Clarendon Press, Oxford, 1977.

2. C. T. H. BAKER, "Methods for Volterra equations of first kind," in Numerical Solution of Integral Equations (L. M. Delves and J. Walsh, eds.), Clarendon Press, Oxford, 1974, pp. 162-174.

3. C. T. H. Baker, A. Makroglou \& E. ShORT, "Regions of stability in the numerical treatment of Volterra integro-differential equations," SIAM J. Numer. Anal., v. 16, 1979, pp. 890-910.

4. H. BrunNer, E. HAIRER \& S. P. NøRSETT, "Runge-Kutta theory for Volterra integral equations of the second kind," Math. Comp., v. 39, 1982, pp. 147-163.

5. L. GAREY, "Solving nonlinear second kind Volterra equations by modified increment methods," SIAM J. Numer. Anal., v. 12, 1975, pp. 501-508. 
6. C. J. Gladwin, "Quadrature rule methods for Volterra integral equations of the first kind," Math. Comp., v. 33, 1979, pp. 705-716.

7. P. Henrici, Discrete Variable Methods in Ordinary Differential Equations, Wiley, New York, 1962.

8. P. J. VAN DER Houwen \& H. J. J. TE RiEle, "Backward differentiation type formulas for Volterra integral equations of the second kind," Numer. Math., v. 37, 1981, pp. 205-217.

9. P. J. VAN DER HOUWEN \& H. J. J. TE RIELE, "Linear multistep methods for Volterra integral equations of the second kind," in: C. T. H. BAKER \& G. F. Miller, Treatment of Integral Equations by. Numerical Methods, Proceedings of a Symposium held at Durham, U. K., July 1982, Academic Press, 1982, pp. 79-94.

10. M. Kobayasi, "On the numerical solution of Volterra integral equations of the second kind by linear multistep methods," Rep. Statist. Appl. Res. Un. Japan. Sci. Engrs., v. 13, 1966, pp. 1-21.

11. J. D. LAmbert, Computational Methods in Ordinary Differential Equations, Wiley, London, 1973.

12. P. Linz, The Numerical Solution of Volterra Integral Equations by Finite Difference Methods, MRC Tech. Summary Report \#825, Math. Research Center, Madison, Wisc., November 1967.

13. P. Linz, "Linear multistep methods for Volterra integro-differential equations," J. Assoc. Comput. Mach., v. 16, 1969, pp. 295-301.

14. A. MaKrogloU, "Convergence of a block-by-block method for nonlinear Volterra integro-differential equations," Math. Comp., v. 35, 1980, pp. 783-796.

15. W. L. MocarSKy, "Convergence of step-by-step methods for nonlinear Volterra integro-differential equations,” J. Inst. Math. Appl., v. 8, 1971, pp. 235-239.

16. B. Noble, A Bibliography on: Methods for Solving Integral Equations, -A Author Listing, MRC Techn. Summary Report. \#1176, Sept. 1971, - Subject Listing, MRC Techn. Summary Report \# 1177, Sept. 1971, Math. Research Center, Madison, Wisc.

17. B. NOBLE, "Instability when solving Volterra integral equations of the second kind by multistep methods," in Conference on the Numerical Solution of Differential Equations (J. L1. Morris, ed.), Lecture Notes in Math., Vol. 109, Springer-Verlag, Berlin, 1969.

18. B. Noble, “The numerical solution of nonlinear integral equations and related topics," in Nonlinear Integral Equations (P. M. Anselone, ed.), Univ. of Wisconsin, Madison, 1964, pp. 215-318.

19. H. J. J. TE RiELE, "Collocation methods for weakly singular second-kind Volterra integral equations with non-smooth solution," IMA J. Numer. Anal., v. 2, 1982, pp. 437-449.

20. P. H. M. Wolkenfelt, "Reducible quadrature methods for Volterra integral equations of the first kind," BIT, v. 21, 1981, pp. 232-241.

21. P. H. M. Wolkenfelt, "The construction of reducible quadrature rules for Volterra integral and integro-differential equations," IMA J. Numer. Anal., v. 2, 1982, pp. 131-152.

22. P. H. M. Wolkenfelt, "Modified multilag methods for Volterra functional equations," Math. Comp., v. 40, 1983, pp. 301-316.

23. P. H. M. Wolkenfelt, P. J. van der Houwen \& C. T. H. Baker, "Analysis of numerical methods for second kind Volterra equations by imbedding techniques," J. Integral Equations, v. 3, 1981, pp. 61-82. 